\title{
Investir avec un privilège fiscal
}

\section{Utilisez les avantages des primes uniques}

Recherchez-vous une opportunité de placement avec un rendement garanti et des avantages fiscaux? Examinez à chaque fois les offres des primes uniques de la FMH Insurance Services!

Dans notre activité de conseil, nous rencontrons souvent des clients qui ont placé dans des obligations à long terme. Le rendement brut paraît être séduisant pour cette forme d'investissement, cependant nous conseillons dans la plupart des cas à nos clients de conclure une prime unique. L'exemple suivant illustre comment nos clients profitent des avantages fiscaux et ainsi améliorent sensiblement leur rendement net.

\section{Exemple chiffré}

Homme, âge 52, durée 8 ans

$\begin{array}{lr}\text { Prime unique } \\ \text { Fr. } & 75000 \\ & - \\ \text { Fr. } & 83522 \\ \text { Fr. } & 7581 \\ \text { Fr. } & 91103 \\ \text { Fr. } & 0 \\ \text { Fr. } & 91103 \\ \text { Fr. } & 16103\end{array}$

\begin{tabular}{lr}
\multicolumn{2}{l}{ Obligation d'Etat * } \\
Fr. & 75000 \\
Fr. & 150 \\
Fr. & 2250 \\
Fr. & 75000 \\
& - \\
Fr. & 92388 \\
Fr. & 6086 \\
Fr. & 86302 \\
Fr. & $\mathbf{1 1 3 0 2}$
\end{tabular}

Placement net

Frais de dépôt par an 0,2\%

Versement de l'intérêt brut annuel

Versement garanti après 8 ans

Parts excédentaires prévues

Versement total probable y compris les intérêts composés

./. impôts (35\% taux marginal)

Valeur totale de votre placement après 8 ans et impôts

Votre gain

Fr. $\quad 16103$

Fr. $\quad 11302$

Le gain engendré par la prime unique par rapport à l'Obligation de la Confédération s'élève à Fr. 4801.-, ce qui correspond à un rendement de plus $42 \%$. * Taux d'intérêt $3 \%$, intérêt de réinvestissement $1 \%$.

\section{Offre spéciale}

Les offres de primes uniques sont nombreuses. Nous analysons continuellement le marché pour trouver les meilleures propositions. Grâce à notre nombreuse clientèle nous pouvons souvent profiter d'offres spéciales à des conditions très avantageuses. Testez notre palette d'offres, nous vous calculons volontiers une offre personnelle.

\section{Talon réponse}

Prière d'envoyer ou de faxer au: 0319595010

Prénom/Nom

Adresse

NPA/Lieu

Date de naissance

Téléphone privé / cabinet

Atteignable le plus facilement (heure)

Adresse e-mail

Prière de $m^{\prime}$ envoyer une offre pour une prime unique.

Montant du placement (min. Fr. 20000)

Durée du placement (min. 5 ans, min. jusqu'à 60 ans)

Prière de me téléphoner pour un conseil personnalisé.

Je m'intéresse à:
Caisse maladie
$3^{\mathrm{e}}$ pilier lié a
Caisse de pension LPP
Planification financière
Optimalisation fiscale
Responsabilité civile prof.

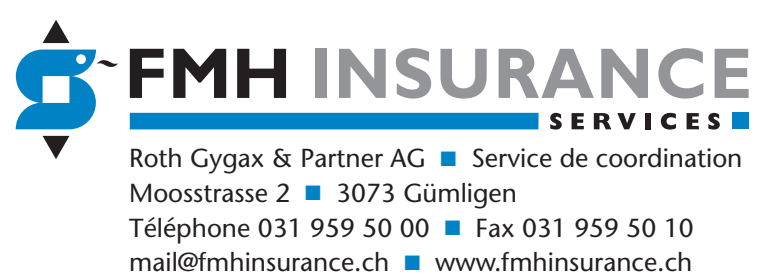

